BMJ Open Sport \& Exercise Medicine

\section{How does the situation before a tackle influence a tackler's head placement in rugby union?: application of the decision tree analysis}

To cite: Suzuki K, Nagai S, Iwai $\mathrm{K}$, et al. How does the situation before a tackle influence a tackler's head placement in rugby union? application of the decision tree analysis. BMJ Open Sport \& Exercise Medicine 2021;7:e000949. doi:10.1136/ bmjsem-2020-000949

Accepted 8 March 202
Check for updates

(c) Author(s) (or their employer(s)) 2021. Re-use permitted under CC BY. Published by BMJ.

${ }^{1}$ Sports Research and Development Core, University of Tsukuba, Tsukuba, Japan ${ }^{2}$ Department of Physical Therapy, Faculty of Health Sciences, Tsukuba International University, Tsuchiura, Ibaraki, Japan

${ }^{3}$ Center of Humanities and Sciences, Ibaraki Prefectural University of Health Sciences, Inashiki-gun, Ibaraki, Japan ${ }^{4}$ Faculty of Health and Sport Sciences, University of Tsukuba, Tsukuba, Ibaraki, Japan

Correspondence to Dr Keita Suzuki; suzuki.keita.wu@alumni. tsukuba.ac.jp

\section{ABSTRACT}

Objectives Tacklers need to decide where to place their head based on the evasive manoeuvres of the ball-carrier and positional relationship with the ballcarrier before tackle. Therefore, it is difficult for tacklers to improve incorrect head placement at the moment of contact. Moreover, the characteristics prior to tackle have a relationship with the tackler's head placement. However, how situations lead to incorrect head placement remains unknown. The aim of this study was to identify pre-contact situations that lead to incorrect head placement by using decision tree analysis.

Methods Tackles leading to concussions were used to identify events that provoked injury using the video recordings of matches. Injury-free tackle was used as a control. All tackles were classified according to head placements and coded from seven pre-contact factors configured aspect of both tacklers and ball-carriers.

Results Three situations that led to incorrect head placement were identified. Evasive manoeuvres implemented by the ball-carrier significantly contributed to the head placement at the time of contact.

Conclusion Our findings suggest that tacklers should keep their heads up to identify the movements of the ball-carrier, which might lead to tackling the head on the correct side at the moment of tackling and decrease the risk of tackler-related concussions.

\section{INTRODUCTION}

Video analysis of tackle-related injuries, ${ }^{1-5}$ including concussions, ${ }^{6-14}$ neck injuries ${ }^{15}$ and shoulder injuries, ${ }^{16}$ has been conducted in rugby players at various competitive levels. In addition, head impact tackle scenes ${ }^{17} 18$ and tackle performance ${ }^{1920}$ have been analysed using video footage. The risk of tackle-related injuries tends to increase when the tackler's head is in front of the ball-carrier. ${ }^{37-9} 1516$ In addition, our previous study showed that the likelihood of concussion increased when the head of the tackler was placed at one side of the ball-carrier. ${ }^{8}$ For a side-on or oblique tackle, the tackler's head on the side of the

\section{What are the new findings?}

- The probability of tackler-related concussions in creased when tackles were attempted with incorrect head placement.

- Three situations before contact were associated with a greater frequency of tackles with incorrect head placement.

- Evasive manoeuvres by the ball-carrier also contributed to the head placement at the time of contact.

- The frequency of the tackle with a correct head placement increased when the tackler faced the ball-carrier or the tackler's head moved in an attempt to follow the ball-carrier.

ball-carrier may be not the correct head position; however, for a front-on tackle, the tackler's head on the side of the ball-carrier is correct. In other words, it may not be sufficient to classify the tackler's head placement with respect to the ball-carrier. Sobue et al defined an incorrect head placement as that with the tackler's head in front of the ball-carrier, which has been mentioned as a risk for the occurrence of concussions. ${ }^{21}$ However, the effect of other head placements (lateral or posterior to the ball-carrier) on the occurrence of the tackler-related concussion have not been clarified. Therefore, in the present study, an incorrect head placement was defined as satisfying either (1) when the tackler's head was placed in front of the ball-carrier (figure $1 \mathrm{~A}-\mathrm{C}$ ) ${ }^{21}$ or (2) when any tackles gave an impact force to the tackler's head from the diagonal, lateral or posterior direction (figure 1D-F).

The characteristics of both the tackler and ball-carrier prior to tackle are associated with the occurrence of tackler-related concussion. Regarding the tackler, whether the tackler is accelerating, ${ }^{6} 13$ looking forward with face raised, ${ }^{8}{ }^{9}$ recognising the ball-carrier, ${ }^{9}$ shortening steps ${ }^{914}$ and the tackler's posture before 


\section{A}

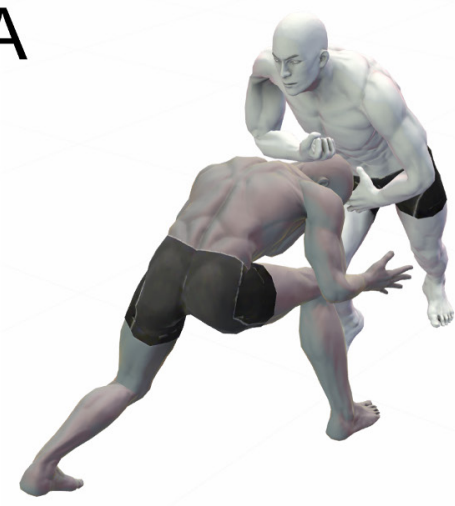

B

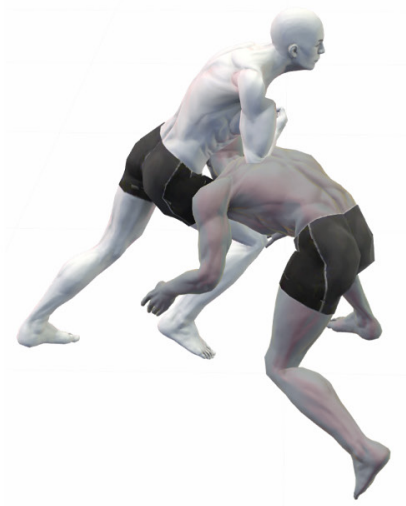

C

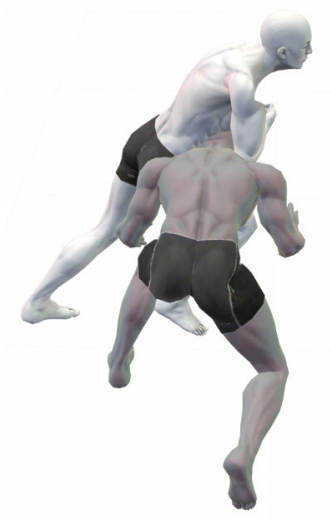

D

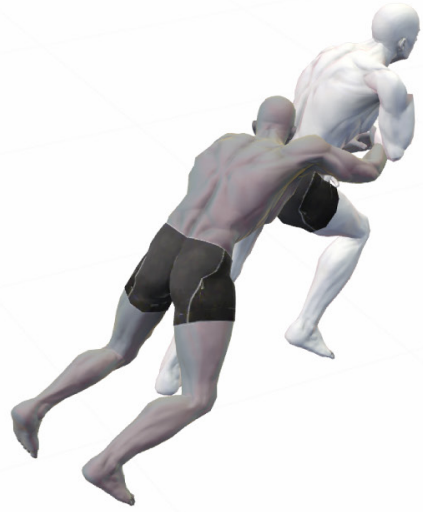

E

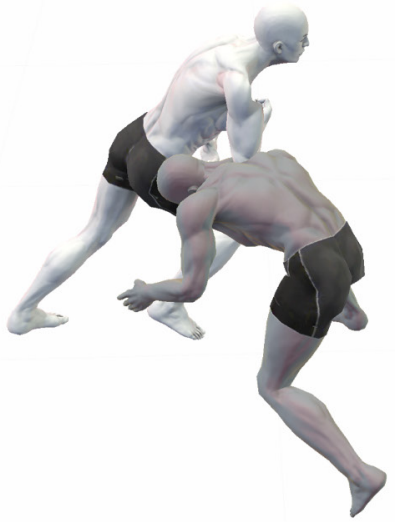

F

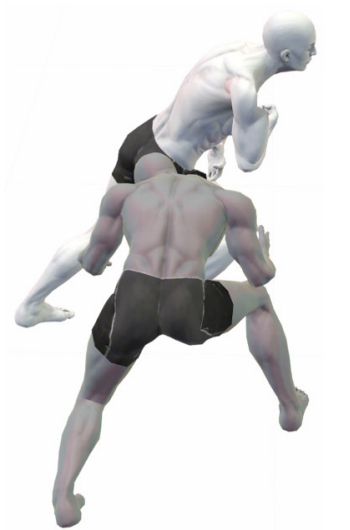

Figure 1 Definition of the incorrect tackler's head placement. (A) The tackler's head is in front of the ball-carrier during a fronton tackle. (B) The tackler's head is in front of the ball-carrier during a tackle from oblique. (C) The tackler's head is in front of the ball-carrier during a side-on tackle. (D) The tackler's head is behind the ball-carrier during a tackle from behind. (E) The tackler's head is placed at one side of the ball-carrier during a tackle from oblique. (F) The tackler's head is placed at one side of the ball-carrier during a side-on tackle. * Grey, a tackler; white, a ball-carrier.

the tackle ${ }^{13}$ are related to the occurrence of tacklerrelated concussion. In the aspect of the ball-carrier, an accelerating ball-carrier ${ }^{613}$ and evasive manoeuvre prior to tackle $^{8}$ have a relationship with the occurrence of tackler-related concussion. In addition, the latest systematic scoping review reported that 'head up and facing forward' and 'shortening steps' were associated with injury prevention and tackling performance in rugby union. ${ }^{22}$ Therefore, tacklers need to decide where to place their own head based on the evasive manoeuvres of the ball-carrier and positional relationship with the ball-carrier before tackle. Thus, it is difficult for tacklers to improve incorrect head placement at the moment of contact. In other words, situations prior to tackling might have a relationship with the tackler's head placement, which is a risk of tackle-related injuries, including concussions. However, coaches often focus attention on the contact phase during tackle-technique training while excluding the pre-contact phase. ${ }^{23}$

It is necessary to identify the situations leading to the main outcome in order to clarify the relationship between the combination of multiple factors and the main outcome. In addition, we believe that it is important that the results of research be easily understood by coaches and players in order to apply them in their coaching and training. Therefore, we focused on decision tree analysis, 
a machine learning technique that has recently been used in the field of sports science. Decision tree analysis, a machine learning technique, has been used to explain certain situations in one-versus-one player interactions, ${ }^{24}$ match outcomes,${ }^{25-28}$ and the effectiveness of ball-screens and inside-passes in basketball. ${ }^{29}{ }^{30}$ Therefore, applying decision tree analysis could reveal pre-contact situations involving the tackler's head placement. The present study aimed to identify the pre-contact situations that lead to incorrect head placement by using decision tree analysis.

\section{METHODS}

\section{Concussion data}

The present study was a case-control study. Injury surveillance of a Japanese collegiate rugby union team was conducted between 2008 and 2015 with informed consent from the players. ${ }^{8}$ There was no patient or public involvement.

In all playing seasons, medically trained personnel from teams recorded injuries according to the consensus statement for injury surveillance. ${ }^{31}$ Furthermore, all players who sustained a head impact or who sustained a suspected concussion during matches were screened by a team doctor in accordance with the Concussion Guidance published by the World Rugby. ${ }^{32}$ All suspected or confirmed concussions were analysed in the present study. ${ }^{33}$

\section{Tackle data}

A tackle has been defined as any event in which one or more tacklers attempt to stop or impede the ballcarrier, whether or not the ball-carrier was brought to the ground. ${ }^{3}$ We used data from our previous study that collected tackles leading and not leading to concussions. ${ }^{8}$ These data included tackles from 34 collegiate rugby union team matches recorded between 2008 and 2015, in which the tacklers sustained concussions. We selected 538 tackles, 34 of which led to concussions (experimental group) and 504 did not result in any type of injury (control group). An a priori power analysis indicated that an estimated total sample size of 208 events, including both concussion and non-injury events, would be required to achieve a power $(1-\beta)$ of 0.80 with a confidence level of $95 \%(\alpha=0.05)$, while an OR of 1.50 is required to produce the smallest worthwhile effect.

\section{Data analysis}

We defined an incorrect head placement as satisfying either (1) when the tackler's head was in front of the ballcarrier (figure $1 \mathrm{~A}-\mathrm{C}$ ) ${ }^{21}$ or (2) when any tackles gave an impact force to the tackler's head contacting from the diagonal, lateral or posterior direction (figure 1D-F). The first author (KS) coded all tackles described previously from seven factors, which configured the aspect of both the tackler and ball-carrier during the pre-contact phase based on both consensus statement on video analysis in rugby union and previous studies (table 1). ${ }^{81934-36}$ Tackles were excluded when the placement of the tackler's head at the time of contact could not be identified. According to our previous research, we obtained high inter-rater reliability between the two raters. ${ }^{37}$ The $\kappa$ statistic result for inter-tester reliability was 0.85 (pre-contact), indicating excellent agreement. ${ }^{38}$

\section{Statistical analysis}

When we used the tackler's head placement as the main outcome of the decision tree analysis, we needed to clarify whether it is also related to concussion in this cohort. Therefore, we calculated OR and 95\% CIs from the results of the binomial logistic regression analysis. If the OR was $>1.0$, concussion was more likely to occur compared with correct head placement. We also recorded situations in pre-contact features that led to incorrect head placement using the decision tree within the rpart package ${ }^{39}$ using $R$ V.3.5.3 (R Foundation for Statistical Computing, Vienna, Austria). The rpart package uses the classification and regression tree (CART) algorithm to build a tree model. In CART, some variables merge such that there are always two branches based on the Gini indexes.

\section{Patient and public involvement}

Patients and/or the public were not involved in the design, or conduct, or reporting, or dissemination plans of this research.

\section{RESULTS}

After excluding 49 tackles that could not be identified with the placement of the tackler's head at the time of contact, we analysed data from 489 tackles (90.9\%), including 34 that led to a concussion $(100 \%)$ and 455 that did not result in any type of injury $(90.2 \%)$. Of these, we classified head placements in 140 and 349 tackles as incorrect and correct, respectively.

Effects of incorrect head placement on the risk of concussion First, we confirmed whether the tackler's head placement was related to concussion by logistic regression analysis in this cohort, in order to use that binary variable as the main outcome of the decision tree analysis. The risk of concussion was significantly higher in tackles with incorrect head placement (OR 8.21; 95\% CI 3.73 to 18.11; $\mathrm{p}<0.01$; table 2). In addition, we assigned tackles with incorrect head placements to groups depending on when the tackler's head was in front of the ball-carrier or when any tackles gave an impact force to the tackler's head contacting from the diagonal, lateral or posterior direction. The risk of concussion increased when the tackler's head was in front (OR 14.78; 95\% CI 6.27 to 34.84; $\mathrm{p}<0.01$; table 2 ), and when any tackles gave impact force to the tackler's head contacting from the diagonal, lateral or posterior direction (OR 3.83; 95\% CI 1.38 to 10.64; $\mathrm{p}=0.01$; table 2) with the ball-carrier compared with the correct head placement. 
Table 1 Characteristics of pre-contact phase variable and their descriptions

\begin{tabular}{|c|c|}
\hline Categorical variables & Descriptions \\
\hline \multicolumn{2}{|l|}{ Tackler } \\
\hline \multicolumn{2}{|l|}{ Stance $^{19}$} \\
\hline Flat footed & Tackler standing square with feet aligned and flat on the ground \\
\hline Back foot & Tackler stepping backwards as ball-carrier approaches \\
\hline Split forward & Tackler standing with staggered stance \\
\hline No stance & Tackler diving or sliding into contact \\
\hline \multicolumn{2}{|c|}{ Direction of movement of tackler ${ }^{819}$} \\
\hline Forward & Toward the ball-carrier \\
\hline Backwards & Back pedalling, that is, away a ball-carrier \\
\hline Lateral & Towards the touchline (across the field) \\
\hline No movement & Tackler did not move before tackle situation \\
\hline \multicolumn{2}{|l|}{ Head position ${ }^{1936}$} \\
\hline Up and forward & Towards ball-carrier \\
\hline Away & Away from ball-carrier \\
\hline Down & Towards the ground \\
\hline In motion & Tackler's head was moving in attempt to follow the ball-carrier \\
\hline \multicolumn{2}{|l|}{ Speed of tackler ${ }^{34} 36$} \\
\hline Fast & Running/sprinting (purposeful running with maximal effort, high knee lift) \\
\hline Moderate & Jogging (non-purposeful slow running with low knee lift) \\
\hline Slow & Stationary/walking (no or few visible foot movement) \\
\hline \multicolumn{2}{|c|}{ Orientation of tackler in relation to ball-carrier ${ }^{19}$} \\
\hline In front & Tackler and ball-carrier moving head on towards each other \\
\hline Side & Tackler moving in from the ball-carrier's side \\
\hline Oblique & Tackler moving into ball-carrier at an angle \\
\hline Behind & Tackler chasing ball-carrier towards own try-line \\
\hline \multicolumn{2}{|l|}{ Ball-carrier } \\
\hline \multicolumn{2}{|c|}{ Speed of ball-carrier ${ }^{34} 36$} \\
\hline Fast & Running/sprinting (purposeful running with maximal effort, high knee lift) \\
\hline Moderate & Jogging (non-purposeful slow running with low knee lift) \\
\hline Slow & Stationary/walking (no or few visible foot movement) \\
\hline \multicolumn{2}{|c|}{ Evasive manoeuvre performed by ball-carrier 83536} \\
\hline Straight run & Ball-carrier running straight at the defence or goal-line \\
\hline Side-step & Ball-carrier performing an evasive step initiated by either leg \\
\hline Arcing run & Ball-carrier performing arcing run \\
\hline Lateral run & Ball-carrier performing a run from touchline to touchline \\
\hline Diagonal run & Ball-carrier running at an angle, instead of straight at the tackler \\
\hline Be tackled & Ball-carrier being tackled by the other tacklers and no movement in any direction \\
\hline No movement & Ball-carrier not moving before tackle situation \\
\hline
\end{tabular}

\section{Situations before contact leading to tackles with incorrect head placement}

Figure 2 shows the features associated with a higher probability of tackling an incorrect head placement at the time of contact. The frequency of tackles with an incorrect head placement was $60.0 \%$ when a ball-carrier side-stepped, ran an arc or was tackled by others, and when the tackler's head was angled downward.
The frequency of an incorrect head placement was also $60.0 \%$ when ball-carriers ran straight or diagonally towards the defence line, when they did not move, or moved at high or low speed; and when the tackler moved laterally, stood with both feet flat on the ground or was located obliquely to the ball-carrier.

Evasive manoeuvres and speed of the ball-carrier were the same as the second set of features. Furthermore, the 
Table 2 Tackler head placements during contact are associated with the tackler-related concussion occurrence

\begin{tabular}{|c|c|c|c|}
\hline $\begin{array}{l}\text { Head placements (vs } \\
\text { no injuries) }\end{array}$ & OR & $(95 \% \mathrm{Cl})$ & $P$ value \\
\hline Correct & Reference & & \\
\hline Incorrect* & 8.21 & (3.73 to 18.11 ) & $<0.01$ \\
\hline Correct & Reference & & \\
\hline Incorrect (1)† & 14.78 & (6.27 to 34.84$)$ & $<0.01$ \\
\hline Incorrect (2)‡ & 3.83 & (1.38 to 10.64 ) & 0.01 \\
\hline
\end{tabular}

${ }^{*}$ An incorrect head placement as satisfying either (1) when the tackler's head was in front of the ball-carrier or (2) when any tackles gave an impact force to the tackler's head in the diagonal, lateral or posterior direction.

†An incorrect head placement as satisfying (1) when the tackler's head was in front of the ball-carrier.

$\ddagger$ An incorrect head placement as satisfying (2) when any tackles gave an impact force to the tackler's head contacting from the diagonal, lateral or posterior direction.

$\mathrm{Cl}$, confidence intervals; OR, odds ratio.

frequency of tackles with an incorrect head placement was $61.9 \%$ when the tacklers moved forward, moved at high or low speed, and were located in front of, next to or behind the ball-carrier.

\section{DISCUSSION}

This is the first attempt to identify the situations before contact leading to tackles with incorrect head placement by using decision tree analysis. To the best of our knowledge, no research on tackle has applied decision tree analysis in the rugby union. Thus, the present study is the first to present pre-contact situations, which configured the aspect of both tackler and ball-carrier, related to the tackler's head placement.

\section{Relationship between incorrect head placement and} concussion

The risk of tackler-related concussion increased 8.2fold with tackles with incorrect head placement, which concurred with some previous findings. ${ }^{7-9}{ }^{21}$ In addition to the traditional definition of incorrect head placement, when the tackler's head was in front of the ball-carrier, we also analysed an incorrect head placement when a tackler's head was in a position of contact with other players without in front of the ball-carrier. As a result, the risk of concussion was significantly increased (OR 3.83) even after excluding tackles with the head in front of the ball-carrier. Rugby Ready, which is a website intended to raise awareness of good practice and help stakeholders manage the inherent risks of contact by the World Rugby, advises positioning the head behind or to one side, and never in front of the ball-carrier in all tackle situations. ${ }^{40}$ Our results provide support for this recommendation by demonstrating the combined effects of head placement of the tackler and direction of the tackle.

\section{Situations leading to the placement of the tackler's head at the time of contact}

Evasive manoeuvres implemented by the ball-carrier significantly contributed to the head placement at the time of contact. The probability of tackles with incorrect head placement also increases when a ball-carrier tries to avoid the tackle by side-stepping and running an arc, or when the tackler's head faces downward. Conversely, even if the evasive manoeuvre of the ball-carrier was the same, the frequency of the tackle with a correct head placement increased when the tackler faced toward the ball-carrier or tackler's head was moving in an attempt

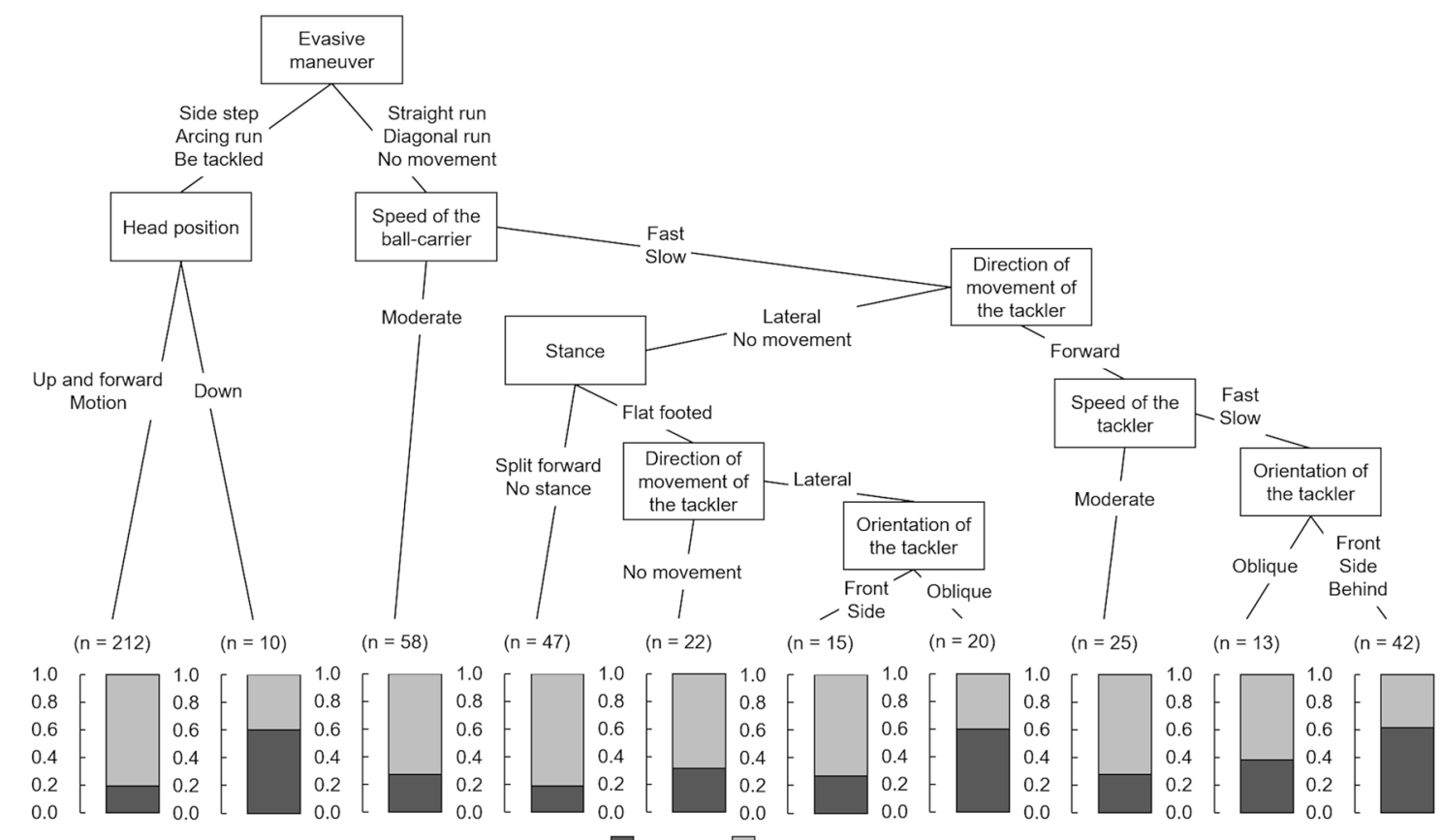

Figure 2 Decision tree according to tackler head placements. 
to follow the ball-carrier. At a professional level, the characteristics 'head up and forward/face up' and 'identify/track ball-carrier onto shoulder' had a lower propensity to result in a head injury assessment for tacklers. ${ }^{9}$ Further, lowered tackler's head increased the frequency of tackler's primary anterior shoulder dislocation. ${ }^{16}$ Moreover, in terms of tackle performance, having the head up, looking forward and facing the ballcarrier before contact affected successful outcomes. ${ }^{19} 20$ Therefore, our results suggest that tacklers should keep their heads up to identify the movements of the ballcarrier, which might lead to tackling the head on the correct side at the moment of tackling and decrease the likelihood of tackler-related injuries and tackle failure.

In addition, when the ball-carrier ran straight to the defence line at high speed, the likelihood of tackling an incorrect head placement increased whether a tackler moved at high or low speeds. When both the tackler and the ball-carrier sprinted, the estimated contact time was shortened, which would interfere with the ability of the tacklers to control their own movements at the time of contact. Conversely, the risk of tackles with incorrect head placements decreased in the same situation mentioned previously, even when a tackler moved at a moderate speed. Therefore, these results suggest that controlling the speed of tacklers to match the movements of ball-carriers is essential. For example, shortening steps to control speed might help to prevent tacklers from placing incorrect head positions and improve tackle performance. 'Shortening steps' was significant in enabling successful tackles ${ }^{20}$ and decreasing the likelihood tackle-related injuries. ${ }^{2} 14$

In the present study, we suggested that the two aforementioned characteristics, 'head up' and 'controlling the tackler's speed', decrease the probability of incorrect head placement during the tackle. We applied the decision tree analysis for clarifying the situations that led to tackler's head placement. As a result, we identified the situations that led to both tackling correct head placement and incorrect head placement. We believe that analysis of the situations that decreased the risk of concussion might lead to better recommendations for the prevention of concussion.

\section{Applying decision tree analysis}

In the present study, we clarified the relationship between a tackler's head placement (the main outcome) and the pre-contact situations of a tackler and the ball-carrier by decision tree analysis. We believe that the decision tree analysis is an approach that allows players, coaches and trainers to visually understand the relationship between the main outcome and multiple factors. Our results showed that the probability of tackles with correct head placement increased regardless of the direction of the tackle when a ball-carrier tries to avoid the tackle by side-stepping, running an arc, and when a tackler faced toward the ball-carrier or tackler's head was moving in an attempt to follow the ball-carrier. When players, especially beginners, improve their interpersonal tackle technique by restricting the ball-carrier's movement and keeping the tackler's head up to check the ball-carrier's movements, they will contribute to training under safe conditions with a lower probability of concussions.

Moreover, this is the first study to adapt decision tree analysis to video analysis of tackling scenes in rugby union. The results showed that a combination of precontact characteristics influenced the head placement of the tackler. Therefore, we believe that new insights can be identified by adapting decision tree analysis to other tackle-related injury mechanisms and tackle performance.

\section{Limitations}

The present study generated important information, and several limitations should be addressed. At first, our study analysed isolated characteristics in the pre-contact phase, which were not involved in any other players or the ground area occurred a tackle. As concussions occur after contact, our results do not directly influence the occurrence of concussions. Therefore, future studies need to determine the effects of concussion by combining the phases in which concussions occur with some of the situations identified in this study. Second, since our results were based on a single collegiate rugby union team, caution should be exercised when generalising our findings, even at the same playing level. Other contextual factors, such as match time and match status at the time of tackles, were not analysed. Tierney et al reported that most direct impacts to the heads of tacklers occur during the second half of matches. ${ }^{18}$ Therefore, the characteristics of tackles with incorrect head placements might differ significantly according to the amount of time spent in matches.

\section{CONCLUSIONS}

We demonstrated the effects of incorrect tackler head placements and the direction of tackles on the risk of tackler-related concussion and identified three situations before contact, which led to tackling incorrect head placement. The risk of tackler-related concussions increased when tackles were attempted with incorrect head placement. Evasive manoeuvres by the ball-carrier also contributed to the head placement at the time of contact. Therefore, our results suggest that tacklers should keep their heads up to identify the movements of the ball-carrier, which might lead to tackling the head on the correct side at the moment of tackling and decreasing the risk of tackler-related concussion.

\section{Twitter Keita Suzuki @K Suzuki0312}

Acknowledgements We thank the players at the University of Tsukuba Rugby Football Club for their participation and cooperation in the study. We are also grateful to the medical staff at the University of Tsukuba Rugby Football Club for recording the match injuries. This work was supported by JSPS KAKENHI Grant Number 19 K20009. 
Contributors KS carried out the study and was directly involved in the conception of the study, collected the injury data, completed the data analysis and drafted the manuscript. SN was involved in collecting the injury data, data analysis and drafting of the manuscript. Kl assisted with data analysis, particularly statistical analysis. TF was involved in collecting match video records. MT was directly involved in the conception of the study and assisted with the draft of the manuscript. All authors have read and approved the final version of the manuscript, and all agree with the order of presentation of the authors.

Funding This work was supported by JSPS KAKENHI Grant Number 19 K20009.

Competing interests None declared.

Patient consent for publication Not required.

Ethics approval The Ethics Committee of the Faculty of Health and Sport Science at the University of Tsukuba approved the present study (reference no. 27-88).

Provenance and peer review Not commissioned; externally peer reviewed.

Data availability statement Data are available on reasonable request. Data are available on reasonable request from KS (suzuki.keita.wu@alumni.tsukuba.ac.jp).

Open access This is an open access article distributed in accordance with the Creative Commons Attribution 4.0 Unported (CC BY 4.0) license, which permits others to copy, redistribute, remix, transform and build upon this work for any purpose, provided the original work is properly cited, a link to the licence is given, and indication of whether changes were made. See: https://creativecommons.org/ licenses/by/4.0/.

\section{ORCID iDs}

Keita Suzuki http://orcid.org/0000-0003-1762-2116

Masahiro Takemura http://orcid.org/0000-0003-4870-9257

\section{REFERENCES}

1 Burger N, Lambert MI, Viljoen W, et al. Mechanisms and factors associated with tackle-related injuries in South African Youth Rugby Union players. Am J Sports Med 2017;45:278-85

2 Burger N, Lambert MI, Viljoen W, et al. Tackle technique and tacklerelated injuries in high-level South African Rugby Union under-18 players: real-match video analysis. Br J Sports Med 2016;50:932-8.

3 Fuller CW, Ashton T, Brooks JHM, et al. Injury risks associated with tackling in rugby union. Br J Sports Med 2010;44:159-67.

4 Mclntosh AS, Savage TN, McCrory P, et al. Tackle characteristics and injury in a cross section of rugby union football. Med Sci Sports Exerc 2010;42:977-84.

5 Quarrie KL, Hopkins WG. Tackle injuries in professional Rugby Union. Am J Sports Med 2008;36:1705-16.

6 Cross MJ, Tucker R, Raftery M, et al. Tackling concussion in professional rugby union: a case-control study of tackle-based risk factors and recommendations for primary prevention. $\mathrm{Br} J$ Sports Med 2019;53:1021-5.

7 Hendricks S, O'Connor S, Lambert M, et al. Video analysis of concussion injury mechanism in under-18 rugby. BMJ Open Sport Exerc Med 2016;2:e000053.

8 Suzuki K, Nagai S, Iwai K, et al. Characteristics and factors of concussion events for tacklers in collegiate rugby union. Scand $J$ Med Sci Sports 2020;30:185-92.

9 Tierney GJ, Denvir K, Farrell G, et al. The effect of tackler technique on head injury assessment risk in elite rugby union. Med Sci Sports Exerc 2018;50:603-8.

10 Tierney GJ, Denvir K, Farrell G, et al. Does ball carrier technique influence tackler head injury assessment risk in elite rugby union? $J$ Sports Sci 2019;37:262-7.

11 Tierney GJ, Simms CK. Can tackle height influence head injury assessment risk in elite rugby union? J Sci Med Sport 2018;21:1210-4.

12 Tucker R, Raftery M, Fuller GW, et al. A video analysis of head injuries satisfying the criteria for a head injury assessment in professional Rugby Union: a prospective cohort study. $\mathrm{Br} J$ Sports Med 2017:51:1147-51.

13 Tucker R, Raftery M, Kemp S, et al. Risk factors for head injury events in professional rugby union: a video analysis of 464 head injury events to inform proposed injury prevention strategies. $\mathrm{Br} \mathrm{J}$ Sports Med 2017;51:1152-7.

14 Hendricks S, O'connor S, Lambert M, et al. Contact technique and concussions in the South African under-18 Coca-Cola Craven Week Rugby tournament. Eur J Sport Sci 2015;15:557-64.
15 Suzuki K, Nagai S, Iwai K, et al. Analysis of tackling events leading to mild cervical injuries in collegiate rugby Union. Int $J$ Sport Health Sci 2020;18:48-56.

16 Maki N, Kawasaki T, Mochizuki T, et al. Video analysis of primary shoulder dislocations in rugby tackles. Orthop J Sports Med 2017;5:232596711771295.

17 Davidow D, Quarrie K, Viljoen W, et al. Tackle technique of rugby union players during head impact tackles compared to injury free tackles. J Sci Med Sport 2018;21:1025-31.

18 Tierney GJ, Lawler J, Denvir K, et al. Risks associated with significant head impact events in elite rugby union. Brain Inj 2016;30:1350-61.

19 Hendricks S, Matthews B, Roode B, et al. Tackler characteristics associated with tackle performance in rugby union. Eur J Sport Sci 2014;14:753-62.

20 Tierney GJ, Denvir K, Farrell G, et al. The effect of technique on tackle gainline success outcomes in elite level rugby union. Int $J$ Sports Sci Coach 2018;13:16-25.

21 Sobue S, Kawasaki T, Hasegawa Y, et al. Tackler's head position relative to the ball carrier is highly correlated with head and neck injuries in rugby. Br J Sports Med 2018;52:353-8.

22 den Hollander S, Ponce C, Lambert M. Tackle and ruck technical proficiency in rugby union and rugby league: a systematic scoping review. Int J Sports Sci Coach 2020.

23 Hendricks S, Lambert M. Tackling in rugby: coaching strategies for effective technique and injury prevention. Int J Sports Sci Coach 2010;5:117-35.

24 Morgan S, Williams MD, Barnes C. Applying decision tree induction for identification of important attributes in one-versus-one player interactions: a hockey exemplar. J Sports Sci 2013;31:1031-7.

25 James LP, Robertson S, Haff GG, et al. Identifying the performance characteristics of a winning outcome in elite mixed martial arts competition. J Sci Med Sport 2017;20:296-301.

26 Leicht AS, Gómez MA, Woods CT. Explaining match outcome during the men's basketball tournament at the Olympic Games. J Sports Sci Med 2017;16:468-73.

27 Robertson S, Back N, Bartlett JD. Explaining match outcome in elite Australian Rules football using team performance indicators. $J$ Sports Sci 2016;34:637-44.

28 Woods CT, Sinclair W, Robertson S. Explaining match outcome and ladder position in the National Rugby League using team performance indicators. J Sci Med Sport 2017;20:1107-11.

29 Courel-Ibáñez J, McRobert AP, Toro EO, et al. Inside pass predicts ball possession effectiveness in NBA basketball. Int J Perform Anal Sport 2016;16:711-25.

30 Gómez Miguel-Ángel, Battaglia O, Lorenzo A, et al. Effectiveness during ball screens in elite basketball games. J Sports Sci 2015;33:1844-52.

31 Fuller CW, Molloy MG, Bagate C, et al. Consensus statement on injury definitions and data collection procedures for studies of injuries in rugby union. Br J Sports Med 2007;41:328-31.

32 Player Welfare. World rugby concussion guidelines. secondary world rugby concussion guidelines, 2014. Available: http://playerwelfare. worldrugby.org/?documentid $=158$

33 Mc Fie S, Brown J, Hendricks S, et al. Incidence and factors associated with concussion injuries at the 2011 to 2014 South African Rugby Union Youth Week Tournaments. Clin J Sport Med 2016;26:398-404.

34 Deutsch MU, Kearney GA, Rehrer NJ. Time-motion analysis of professional rugby union players during match-play. J Sports Sci 2007;25:461-72.

35 Wheeler KW, Sayers MGL. Modification of agility running technique in reaction to a defender in rugby union. $J$ Sports Sci Med 2010:9:445-51.

36 Hendricks S, Till K, den Hollander S, et al. Consensus on a video analysis framework of descriptors and definitions by the Rugby Union Video Analysis Consensus group. Br J Sports Med 2020;54:566-72.

37 Suzuki K, Nagai S, Ogaki R, et al. Video analysis of tackling situations leading to concussion in collegiate rugby union. J Phys Fit Sports Med 2019;8:79-88.

38 Kirkwood BR, Sterne JAC. Essential medical statistics. 2 edn. Oxford: Blackwell Science, 2003.

39 rpart. Recursive partitioning and regression trees [program] 2018.

40 Rugby Ready. Secondary Rugby Ready, 2014. Available: http://www. irbrugbyready.com/? section=65\&language=EN 